\title{
THE LINEAR ALGEBRA IN THE QUATERNIONIC PLURIPOTENTIAL THEORY
}

\author{
WEI WANG
}

\begin{abstract}
We clarify the linear algebra used in the quaternionic pluripotential theory so that proofs of several results there can be greatly simplified. In particular, we characterize and normalize real 2-forms with respect to the quaternionic structure, and show that the Moore determinant of a quaternionic hyperhermitian matrix is the coefficient of the exterior product of the associated real 2-form. As a corollary, the quaternionic Monge-Ampère operator is the coefficient of the exterior product of the Baston operator.
\end{abstract}

\section{INTRODUCTION}

Alesker [1] 2] proved a quaternionic version of Chern-Levine-Nirenberg estimate and extended the definition of the quaternionic Monge-Ampère operator to continuous quaternionic plurisubharmonic functions. The quaternionic Monge-Ampère operator on quaternionic manifolds was introduced and investigated by Alesker, Shelukhin and Verbitsky et. al. [4]-[6]. More generally, it is interesting to develope pluripotential theory on calibrated manifolds [10]-[11. By using the twistor method in complex geometry, Alesker observed [3] (see also [5]) the coincidence between the quaternionic Monge-Ampère operator and the $n$-th exterior power of the Baston operator $\triangle$. The Baston operator $\triangle$ is the first operator of 0-Cauchy-Fueter complex, which is known explicitly [7] [9] [17] [19. Motivated by this fact, we [15] introduced the first-order differential operators $d_{0}$ and $d_{1}$ acting on the quaternionic version of differential forms. The behavior of $d_{0}, d_{1}$ and $\triangle=d_{0} d_{1}$ is very similar to $\partial, \bar{\partial}$ and $\partial \bar{\partial}$ in several complex variables, and several results in the complex pluripotential theory (cf. e.g. [8] [12]) were extended to the quaternionic case [13]-[16]. The purpose of this paper is to clarify the linear algebra used in the quaternionic pluripotential theory in [15] to simplify proofs of several results there.

Denote by $M_{\mathbb{F}}(p, m)$ the space of $\mathbb{F}$-valued $(p \times m)$-matrices, where $\mathbb{F}=\mathbb{R}, \mathbb{C}, \mathbb{H}$. Motivated by the well known embedding of the quaternionic algebra $\mathbb{H}$ into $\operatorname{End}\left(\mathbb{C}^{2}\right)$ given by

$$
x_{0}+\mathbf{i} x_{1}+\mathbf{j} x_{2}+\mathbf{k} x_{3} \mapsto\left(\begin{array}{rr}
x_{0}+\mathbf{i} x_{1} & -x_{2}-\mathbf{i} x_{3} \\
x_{2}-\mathbf{i} x_{3} & x_{0}-\mathbf{i} x_{1}
\end{array}\right),
$$

we define the embedding

$$
\tau: M_{\mathbb{F}}(p, m) \longrightarrow M_{\mathbb{C}}(2 p, 2 m)
$$

Supported by National Nature Science Foundation in China (No. 11571305) .

Department of Mathematics, Zhejiang University, Zhejiang 310027, P. R. China, Email: wwang@zju.edu.cn. 
as follows. For a quaternionic $p \times m$-matrix $\mathcal{M}$, we write $\mathcal{M}=a+b \mathbf{j}$ for some complex matrices $a, b \in M_{\mathbb{C}}(p, m)$. Then $\tau(\mathcal{M})$ is the complex $(2 p \times 2 m)$-matrix

$$
\tau(\mathcal{M}):=\left(\begin{array}{cc}
a & -b \\
\bar{b} & \bar{a}
\end{array}\right) .
$$

Let $\wedge^{2 k} \mathbb{C}^{2 n}$ be the $k$-th exterior product of $\mathbb{C}^{2 n}, k=1, \ldots, n$. There exists a real linear action $\rho(\mathbf{j})$ on $\mathbb{C}^{2 n}$ naturally (2.13), which induces an action on $\wedge^{2 k} \mathbb{C}^{2 n}$. An element $\varphi$ of $\wedge^{2 k} \mathbb{C}^{2 n}$ is called real if $\rho(\mathbf{j}) \varphi=\varphi$. Denote by $\wedge_{\mathbb{R}}^{2 k} \mathbb{C}^{2 n}$ the subspace of all real elements in $\wedge^{2 k} \mathbb{C}^{2 n}$, which is the counterpart of the space of $(k, k)$-forms in complex analysis. Fix a basis $\left\{\omega^{0}, \omega^{1}, \ldots, \omega^{2 n-1}\right\}$ of $\mathbb{C}^{2 n}$. We characterize and normalize real 2-forms as follows.

Theorem 1.1. (1) For a complex skew symmetric matrix $M=\left(M_{A B}\right) \in M_{\mathbb{C}}(2 n, 2 n)$, the 2 -form

$$
\omega=\sum_{A, B=0}^{2 n-1} M_{A B} \omega^{A} \wedge \omega^{B}
$$

is real if and only if there exists a hyperhermitian $n \times n$-matrix $\mathcal{M}=\left(\mathcal{M}_{j k}\right)$, i.e. $\mathcal{M}_{j k}=\overline{\mathcal{M}_{k j}}$, such that

$$
M=\tau(\mathcal{M}) J
$$

where

$$
J=\left(\begin{array}{cc}
0 & I_{n} \\
-I_{n} & 0
\end{array}\right)
$$

(2) When $\omega$ in (1.3) is real, there exists a quaternionic unitary matrix $\mathcal{E} \in U_{\mathbb{H}}(n)$ such that

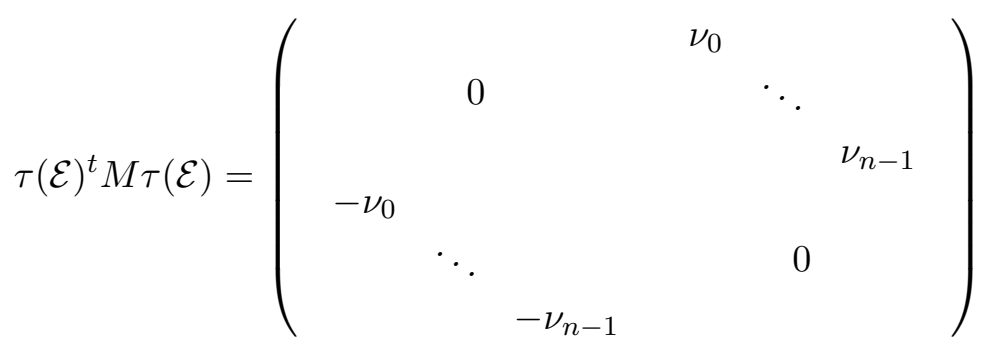

for some real numbers $\nu_{0}, \ldots, \nu_{n-1}$. Namely, we can normalize $\omega$ as

$$
\omega=2 \sum_{l=0}^{n-1} \nu_{l} \widetilde{\omega}^{l} \wedge \widetilde{\omega}^{l+n}
$$

with $\widetilde{\omega}^{A}=\mathcal{E}^{*} . \omega^{A}$. In particular, $\omega$ is strongly positive if and only if $\nu_{l} \geq 0, l=0 \ldots, n-1$, and at least one is positive.

The Moore determinant denoted by det is a determinant defined for quaternionic hyperhermitian matrices and takes real values. Because of the noncommutativity of quaternionic numbers, the Moore determinant is very complicated. Here we find a concrete expression of the Moore determinant of a quaternionic hyperhermitian matrix as follows.

More generally, consider the homogeneous polynomial $\operatorname{det}\left(\lambda_{1} \mathcal{M}_{1}+\ldots+\lambda_{n} \mathcal{M}_{n}\right)$ in real variables $\lambda_{1}, \ldots, \lambda_{n}$ of degree $n$. The coefficient of the monomial $\lambda_{1} \cdots \lambda_{n}$ divided by $n$ ! is called the mixed discriminant of the hyperhermitian matrices $\mathcal{M}_{1}, \ldots, \mathcal{M}_{n}$, and it is denoted 
by $\operatorname{det}\left(\mathcal{M}_{1}, \ldots, \mathcal{M}_{n}\right)$. In particular, when $\mathcal{M}_{1}=\ldots=\mathcal{M}_{n}=\mathcal{M}, \operatorname{det}\left(\mathcal{M}_{1}, \ldots, \mathcal{M}_{n}\right)=$ $\operatorname{det}(\mathcal{M})$. For skew symmetric matrices $M^{(t)} \in M_{\mathbb{C}}(2 n, 2 n), t=1, \ldots, n$, such that 2-forms $\omega_{t}=\sum_{A, B=0}^{2 n-1} M_{A B}^{(t)} \omega^{A} \wedge \omega^{B}$ are real, we define $\triangle_{n}\left(M^{(1)}, \ldots, M^{(n)}\right)$ by

$$
\triangle_{n}\left(M^{(1)}, \ldots, M^{(n)}\right) \Omega_{2 n}:=\omega_{1} \wedge \cdots \wedge \omega_{n},
$$

where

$$
\Omega_{2 n}:=\omega^{0} \wedge \omega^{n} \cdots \wedge \omega^{n-1} \wedge \omega^{2 n-1} \in \wedge_{\mathbb{R}}^{2 n} \mathbb{C}^{2 n} .
$$

Theorem 1.2. For hyperhermitian matrices $\mathcal{M}_{1}, \ldots, \mathcal{M}_{n} \in M_{\mathbb{H}}(n, n)$, we have

$$
2^{n} n ! \operatorname{det}\left(\mathcal{M}_{1}, \ldots, \mathcal{M}_{n}\right)=\triangle_{n}\left(\tau\left(\mathcal{M}_{1}\right) J, \ldots, \tau\left(\mathcal{M}_{n}\right) J\right) .
$$

Alesker introduced in [1] the mixed quaternionic Monge-Ampère operator $\operatorname{det}\left(u_{1}, \ldots, u_{n}\right)$ for real $C^{2}$ functions $u_{1}, \ldots, u_{n}$ as

$$
\operatorname{det}\left(u_{1}, \ldots, u_{n}\right):=\operatorname{det}\left(\left(\frac{\partial^{2} u_{1}}{\partial \overline{q_{l}} \partial q_{k}}(q)\right), \ldots,\left(\frac{\partial^{2} u_{n}}{\partial \overline{q_{l}} \partial q_{k}}(q)\right)\right) .
$$

Recall that for a real $C^{2}$ function $u$, the Baston operator is

$$
\triangle u=\sum_{A, B=0}^{2 n-1} \triangle_{A B} u \omega^{A} \wedge \omega^{B},
$$

where

$$
\triangle_{A B} u:=\frac{1}{2}\left(\nabla_{A 0^{\prime}} \nabla_{B 1^{\prime}} u-\nabla_{B 0^{\prime}} \nabla_{A 1^{\prime}} u\right),
$$

i.e. $2 \triangle_{A B}$ is the determinant of the $(2 \times 2)$-submatrix of $A$-th and $B$-th rows in

$$
\left(\begin{array}{cc}
\nabla_{00^{\prime}} & \nabla_{01^{\prime}} \\
\vdots & \vdots \\
\nabla_{l 0^{\prime}} & \nabla_{l 1^{\prime}} \\
\vdots & \vdots \\
\nabla_{n 0^{\prime}} & \nabla_{n 1^{\prime}} \\
\vdots & \vdots \\
\nabla_{(n+l) 0^{\prime}} & \nabla_{(n+l) 1^{\prime}} \\
\vdots & \vdots
\end{array}\right):=\left(\begin{array}{cc}
\partial_{x_{0}}+\mathbf{i} \partial_{x_{1}} & -\partial_{x_{2}}-\mathbf{i} \partial_{x_{3}} \\
\vdots & \vdots \\
\partial_{x_{4 l}}+\mathbf{i} \partial_{x_{4 l+1}} & -\partial_{x_{4 l+2}}-\mathbf{i} \partial_{x_{4 l+3}} \\
\vdots & \vdots \\
\partial_{x_{2}}-\mathbf{i} \partial_{x_{3}} & \partial_{x_{0}}-\mathbf{i} \partial_{x_{1}} \\
\vdots & \vdots \\
\partial_{x_{4 l+2}}-\mathbf{i} \partial_{x_{4 l+3}} & \partial_{x_{4 l}}-\mathbf{i} \partial_{x_{4 l+1}} \\
\vdots & \vdots
\end{array}\right)
$$

$\nabla_{A \alpha}$ 's above and $\tau(\mathcal{M})$ in (1.2) are relabeling of corresponding notations in [15] (cf. Remark 2.1). We use this relabeling in this paper because it makes some of proofs more transparent.

Theorem 1.3. Let $u_{1}, u_{2}, \ldots, u_{n}$ be real $C^{2}$ functions on $\mathbb{H}^{n}$. Then we have:

$$
\triangle u_{1} \wedge \ldots \wedge \triangle u_{n}=n ! \operatorname{det}\left(u_{1}, u_{2}, \ldots, u_{n}\right) \Omega_{2 n} .
$$

The complexified version of this theorem was proved by Alesker [3] by using the twistor method of complex geometry. The proof given in the appendix (Theorem A.1) of [15] is elementary except for using the fact that the linear combinations of delta functions supported on quaternionic hyperplanes are dense in the space of generalized functions. Here we give a completely elementary proof only by using linear algebra. In Section 2, we prove characterization and normalization 
of real 2-forms in Theorem 1.1, by which we can prove Theorem 1.2. Theorem 1.3 is proved in Section 3. We also give a very simple proof of the fact that $-\frac{1}{\|q\|^{2}}$ is the fundamental solution of the quaternionic Monge-Ampère operator. In Section 4, we give us a simplified proof of the invariance of $d_{\alpha}$ and $\triangle$ under quaternionic linear transformations.

\section{ReAl Forms And the MoOre Determinant}

Denote by $\mathrm{GL}_{\mathbb{H}}(n)$ the set of all invertible quaternionic $(n \times n)$-matrices, and denote by $\mathrm{U}_{\mathbb{H}}(n)$ the set of all unitary quaternionic $(n \times n)$-matrices, i.e.,

$$
\mathrm{U}_{\mathbb{H}}(n)=\left\{\mathcal{M} \in \mathrm{GL}_{\mathbb{H}}(n), \mathcal{M}^{*} \mathcal{M}=\mathcal{M} \mathcal{M}^{*}=I_{n}\right\},
$$

where $\mathcal{M}^{*}=\overline{\mathcal{M}}^{t}$, i.e. $\left(\mathcal{M}^{*}\right)_{j k}=\overline{\mathcal{M}_{k j}}$. Here $\bar{q}=x_{0}-x_{1} \mathbf{i}-x_{2} \mathbf{j}-x_{3} \mathbf{k}$ is the quaternionic conjugate of $q=x_{0}+x_{1} \mathbf{i}+x_{2} \mathbf{j}+x_{3} \mathbf{k}$.

Proposition 2.1. (Claim 1.1.4, 1.1.7 in [1]) For a hyperhermitian $(n \times n)$-matrix $\mathcal{M}$, there exists a unitary matrix $\mathcal{E}$ such that $\mathcal{E}^{*} \mathcal{M E}$ is diagonal and real.

Proposition 2.2. (Theorem 1.1.9 in [1]) (1) The Moore determinant of any complex hermitian matrix considered as a quaternionic hyperhermitian matrix is equal to its usual determinant.

(2) For any quaternionic hyperhermitian $(n \times n)$-matrix $\mathcal{M}$ and any quaternionic $(n \times n)$ matrix $\mathcal{C}$

$$
\operatorname{det}\left(\mathcal{C}^{*} \mathcal{M C}\right)=\operatorname{det}(\mathcal{A}) \operatorname{det}\left(\mathcal{C}^{*} \mathcal{C}\right)
$$

Proposition 2.3. (1) For a quaternionic $(p \times m)$-matrix $\mathcal{M}$ and a quaternionic $(m \times l)$-matrix $\mathcal{N}$, we have

$$
\tau(\mathcal{M N})=\tau(\mathcal{M}) \tau(\mathcal{N})
$$

(2) For $M \in M_{\mathbb{C}}(2 n, 2 n)$, there exists $\mathcal{M} \in M_{\mathbb{H}}(n, n)$ such that $M=\tau(\mathcal{M})$ if and only if

$$
J \bar{M}=M J .
$$

(3) $\tau\left(\mathcal{M}^{*}\right)=\overline{\tau(\mathcal{M})}^{t}$ for any quaternionic $(n \times n)$-matrix $\mathcal{M} . \mathcal{M} \in U_{\mathbb{H}}(n)$ if and only if $\tau(\mathcal{M})$ is complex unitay and symplectic, i.e.

$$
\tau(\mathcal{M}) J \tau(\mathcal{M})^{t}=J
$$

Proof. Write $\mathcal{M}=a+b \mathbf{j}$ as in (1.2). If we write the quaternionic $(m \times l)$-matrix $\mathcal{N}=c+d \mathbf{j}$ for some complex matrices $c, d \in M_{\mathbb{C}}(m, l)$, we have

$$
\mathcal{M N}=(a+b \mathbf{j})(c+d \mathbf{j})=a c-b \bar{d}+(a d+b \bar{c}) \mathbf{j}
$$

by

$$
\mathbf{j} d=\bar{d} \mathbf{j}
$$

Then (2.4) implies

$$
\tau(\mathcal{M N})=\left(\begin{array}{rr}
a c-b \bar{d} & -a d-b \bar{c} \\
\overline{a d}+\bar{b} c & \overline{a c}-\bar{b} d
\end{array}\right)=\left(\begin{array}{rr}
a & -b \\
\bar{b} & \bar{a}
\end{array}\right)\left(\begin{array}{rr}
c & -d \\
\bar{d} & \bar{c}
\end{array}\right)=\tau(\mathcal{M}) \tau(\mathcal{N}),
$$

by definition of $\tau$.

(2) If we write

$$
M=\left(\begin{array}{ll}
a & b \\
c & d
\end{array}\right)
$$


where $a, b, c, d$ are $n \times n$ complex matrices, then (2.2) is equivalent to $c=-\bar{b}, d=\bar{a}$, i.e.

$$
M=\left(\begin{array}{rr}
a & b \\
-\bar{b} & \bar{a}
\end{array}\right) .
$$

Thus $M=\tau(\mathcal{M})$ with $\mathcal{M}=a-b \mathbf{j}$. The converse is also true.

(3) By definition, the quaternionic conjugate of $\mathcal{M}=a+b \mathbf{j}$ is $\overline{\mathcal{M}}=\bar{a}-b \mathbf{j}$. Then we have

$$
\overline{\mathcal{M}}^{t}=\bar{a}^{t}-b^{t} \mathbf{j}
$$

and so

$$
\tau\left(\mathcal{M}^{*}\right)=\left(\begin{array}{cc}
\bar{a}^{t} & b^{t} \\
-\bar{b}^{t} & a^{t}
\end{array}\right)=\overline{\tau(\mathcal{M})}^{t} .
$$

$\tau(\mathcal{M})$ is unitary by $\overline{\tau(\mathcal{M})}^{t} \tau(\mathcal{M})=\tau\left(\mathcal{M}^{*} \mathcal{M}\right)=I_{2 n}$. The symplecticity (2.3) of $\tau(\mathcal{M})$ follows from (2.7) and (2.2). The converse is also true.

Remark 2.1. This Proposition is essentially a conjugate and relabelling version of proposition 2.1 in 15, where $J$ equals to

$$
\left(\begin{array}{ccccccc}
0 & 1 & & & & & \\
-1 & 0 & & & & & \\
& & 0 & 1 & & & \\
& & -1 & 0 & & & \\
& & & & \ddots & & \\
& & & & & 0 & 1 \\
& & & & & -1 & 0
\end{array}\right)
$$

and the embedding $\tau$ maps a quaternionic $(l \times m)$-matrix $\mathcal{A}=\left(\mathcal{A}_{j k}\right)_{l \times m}$ to a complex $(2 l \times 2 m)$ matrices

$$
\tau(\mathcal{A})=\left(\begin{array}{ccc}
\tau\left(\mathcal{A}_{00}\right) & \tau\left(\mathcal{A}_{01}\right) & \ldots \\
\tau\left(\mathcal{A}_{10}\right) & \tau\left(\mathcal{A}_{11}\right) & \ldots \\
\ldots & \ldots & \ddots
\end{array}\right)
$$

Here $\tau\left(\mathcal{A}_{j k}\right)$ is the complex $(2 \times 2)$-matrix

$$
\left(\begin{array}{cc}
a_{j k}^{0}-i a_{j k}^{1} & -a_{j k}^{2}+i a_{j k}^{3} \\
a_{j k}^{2}+i a_{j k}^{3} & a_{j k}^{0}+i a_{j k}^{1}
\end{array}\right)
$$

if we write $\mathcal{A}_{j k}=a_{j k}^{0}+i a_{j k}^{1}+j a_{j k}^{2}+\boldsymbol{k} a_{j k}^{3} \in \mathbb{H}$. One advantage of using $\tau$ as in (1.2) is that the proof of Proposition 2.3 becomes more transparent.

For $M \in M_{\mathbb{C}}(2 n, 2 n)$, define its $\mathbb{C}$-linear action on $\mathbb{C}^{2 n}$ as:

$$
M . \omega^{A}=\sum_{B=0}^{2 n-1} M_{A B} \omega^{B},
$$

and define the induced action on $\wedge^{2 k} \mathbb{C}^{2 n}$ as

$$
M .\left(\omega^{A_{1}} \wedge \ldots \wedge \omega^{A_{2 k}}\right)=M \cdot \omega^{A_{1}} \wedge \ldots \wedge M \cdot \omega^{A_{2 k}} .
$$


For $\mathcal{M} \in M_{\mathbb{H}}(n, n)$, define its induced $\mathbb{C}$-linear action on $\mathbb{C}^{2 n}$ as

$$
\mathcal{M} \cdot \omega^{A}=\tau(\mathcal{M}) \cdot \omega^{A}
$$

and so on $\wedge^{2 k} \mathbb{C}^{2 n}$.

Note that for $\mathcal{M} \in \mathrm{U}_{\mathbb{H}}(n), \mathcal{M} . \beta_{n}=\beta_{n}$ for $\beta_{n}$ given by

$$
\beta_{n}:=\sum_{l=0}^{n-1} \omega^{l} \wedge \omega^{n+l},
$$

since $\tau(\mathcal{M})$ is symplectic by Proposition 2.3 (3) and so

$$
\begin{aligned}
\mathcal{M} . \beta_{n} & =\sum_{l=0}^{n-1} \sum_{A, B} \tau(\mathcal{M})_{l A} \tau(\mathcal{M})_{(n+l) B} \omega^{A} \wedge \omega^{B} \\
& =\frac{1}{2} \sum_{A, B}\left[\tau(\mathcal{M})^{t} J \tau(\mathcal{M})\right]_{A B} \omega^{A} \wedge \omega^{B}=\sum_{l=0}^{n-1} \omega^{l} \wedge \omega^{l+n} .
\end{aligned}
$$

Consequently $\mathcal{M} .\left(\wedge^{n} \beta_{n}\right)=\wedge^{n} \beta_{n}$, i.e.,

$$
\mathcal{M} \cdot \Omega_{2 n}=\Omega_{2 n},
$$

where $\Omega_{2 n}$ is given by (1.9). This means that $\beta_{n}$ and $\Omega_{2 n}$ are invariant under unitary transformations on $\mathbb{H}^{n}$.

Recall that $\mathbf{j}$ induces a real linear map [15]

$$
\rho(\mathbf{j}): \mathbb{C}^{2 n} \rightarrow \mathbb{C}^{2 n}, \quad \rho(\mathbf{j})\left(z \omega^{k}\right)=\bar{z} J \cdot \omega^{k} .
$$

$\mathbf{j}$ is not $\mathbb{C}$-linear, but it is anti-complex linear. It induces an action on $\wedge^{k} \mathbb{C}^{2 n} \cdot \wedge_{\mathbb{R}}^{2 k} \mathbb{C}^{2 n}$ is the subspace of all real elements in $\wedge^{2 k} \mathbb{C}^{2 n}$. $\beta_{n}$ above is real, and so is $\Omega_{2 n}$.

A quaternionic $(m \times k)$-matrix $\mathcal{M} \in M_{\mathbb{H}}(m, k)$ defines a map $\eta: \mathbb{H}^{k} \rightarrow \mathbb{H}^{m}$ given by

$$
\eta\left(q_{1}, \ldots q_{k}\right)=\left(\cdots, \sum_{l=1}^{k} \mathcal{M}_{j l} q_{l}, \cdots\right),
$$

which is right $\mathbb{H}$-linear, i.e. $\eta\left(q_{1} q, \ldots q_{k} q\right)=\eta\left(q_{1}, \ldots q_{k}\right) q$ for any $q \in \mathbb{H}$. It induces a $\mathbb{C}$-linear map $\tau(\mathcal{M}): \mathbb{C}^{2 k} \rightarrow \mathbb{C}^{2 m}$. Similar to $(2.9)$, the induced $\mathbb{C}$-linear pulling back transformation $\eta^{*}: \mathbb{C}^{2 m} \rightarrow \mathbb{C}^{2 k}$ is defined as:

$$
\eta^{*} \widetilde{\omega}^{p}=\sum_{j=0}^{2 k-1} \tau(\mathcal{M})_{p j} \omega^{j}, \quad p=0, \ldots, 2 m-1,
$$

where $\left\{\widetilde{\omega}^{0}, \ldots, \widetilde{\omega}^{2 m-1}\right\}$ is a basis of $\mathbb{C}^{2 m}$ and $\left\{\omega^{0}, \ldots, \omega^{2 k-1}\right\}$ is a basis of $\mathbb{C}^{2 k}$. It induces a $\mathbb{C}$-linear pulling back transformation on $\wedge^{2 k} \mathbb{C}^{2 m}$ given by $\eta^{*}(\alpha \wedge \beta)=\eta^{*} \alpha \wedge \eta^{*} \beta$ inductively.

An element $\omega \in \wedge_{\mathbb{R}}^{2 k} \mathbb{C}^{2 n}$ is said to be elementary strongly positive if there exist linearly independent right $\mathbb{H}$-linear mappings $\eta_{j}: \mathbb{H}^{n} \rightarrow \mathbb{H}, j=1, \ldots, k$, so that

$$
\omega=\eta_{1}^{*} \widetilde{\omega}^{0} \wedge \eta_{1}^{*} \widetilde{\omega}^{1} \wedge \ldots \wedge \eta_{k}^{*} \widetilde{\omega}^{0} \wedge \eta_{k}^{*} \widetilde{\omega}^{1},
$$

where $\left\{\widetilde{\omega}^{0}, \widetilde{\omega}^{1}\right\}$ is a basis of $\mathbb{C}^{2}$. The following corollary easily follows from the definition. 
Corollary 2.1. $\omega \in \wedge_{\mathbb{R}}^{2 k} \mathbb{C}^{2 n}$ is elementary strongly positive if and only if

$$
\omega=\mathcal{M} \cdot\left(\omega^{0} \wedge \omega^{n} \wedge \ldots \wedge \omega^{k-1} \wedge \omega^{n+k-1}\right)
$$

for some quaternionic matrix $\mathcal{M} \in M_{\mathbb{H}}(n)$ with first $k$ rows linearly independent.

An element $\omega \in \wedge_{\mathbb{R}}^{2 k} \mathbb{C}^{2 n}$ is called strongly positive if it belongs to the convex cone $\mathrm{SP}^{2 k} \mathbb{C}^{2 n}$ in $\wedge_{\mathbb{R}}^{2 k} \mathbb{C}^{2 n}$ generated by elementary strongly positive $2 k$-elements. An $2 k$-element $\omega$ is said to be positive if for any elementary strongly positive element $\eta \in \mathrm{SP}^{2 n-2 k} \mathbb{C}^{2 n}, \omega \wedge \eta$ is strongly positive. By definition, $\beta_{n}$ is a strongly positive 2 -form, and $\beta_{n}^{n}=\wedge^{n} \beta_{n}=n ! \Omega_{2 n}$ is a positive $2 n$-form.

Proof of Theorem 1.1. (1) For $\omega$ given by (1.3), note that

$$
\rho(\mathbf{j}) \omega=\sum_{A, B=0}^{2 n-1} \overline{M_{A B}} J \cdot \omega^{A} \wedge J \cdot \omega^{B}=\sum_{C, D=0}^{2 n-1}\left(J^{t} \bar{M} J\right)_{C D} \omega^{C} \wedge \omega^{D}
$$

by definition of $\rho(\mathbf{j})$. So $\omega$ is a real 2 -form if and only if $J^{t} \bar{M} J=M$, i.e.

$$
J \bar{M}=M J
$$

by $J^{2}=-I_{2 n}$ and $J^{t}=-J$. Then by Proposition $2.3(2)$, we can write

$$
M=\left(\begin{array}{cc}
a & -b \\
\bar{b} & \bar{a}
\end{array}\right)
$$

for some complex matrices $a, b \in M_{\mathbb{C}}(n, n)$, and so

$$
M=\left(\begin{array}{rr}
-b & -a \\
\bar{a} & -\bar{b}
\end{array}\right) J=\tau(-b+a \mathbf{j}) J
$$

where $\mathcal{M}:=-b+a \mathbf{j}$ is hyperhermitian. This is because skew-symmetry of $M$ is equivalent to

$$
b^{t}=\bar{b}, \quad a^{t}=-a,
$$

and so

$$
(\overline{-b+a \mathbf{j}})^{t}=(-\bar{b}-a \mathbf{j})^{t}=-b+a \mathbf{j},
$$

where the bar in the left hand side is the quaternionic conjugate.

(2) By applying Proposition 2.1 (1) to the hyperhermitian matrix $\mathcal{M}$, we can find a quaternionic unitary matrix $\mathcal{B}=e+f \mathbf{j} \in U_{\mathbb{H}}(n)$ so that

$$
\overline{\mathcal{B}}^{t}(-b+a \mathbf{j}) \mathcal{B}=\operatorname{diag}\left(\nu_{0}, \ldots, \nu_{n-1}\right)
$$

for some real numbers $\nu_{0}, \ldots, \nu_{n-1}$. Multiply $-\mathbf{j}$ from right on both sides to get

$$
\left(\bar{e}^{t}-f^{t} \mathbf{j}\right)(a+b \mathbf{j})(\bar{e}+\bar{f} \mathbf{j})=\operatorname{diag}\left(-\nu_{1} \mathbf{j}, \ldots,-\nu_{n} \mathbf{j}\right),
$$

by

$$
(e+f \mathbf{j}) \mathbf{j}=\mathbf{j}(\bar{e}+\bar{f} \mathbf{j}) \quad \text { and } \quad-(-b+a \mathbf{j}) \mathbf{j}=a+b \mathbf{j}
$$


by using (2.5). Apply the mapping $\tau$ as a representation (2.1) on both sides of (2.20) to get

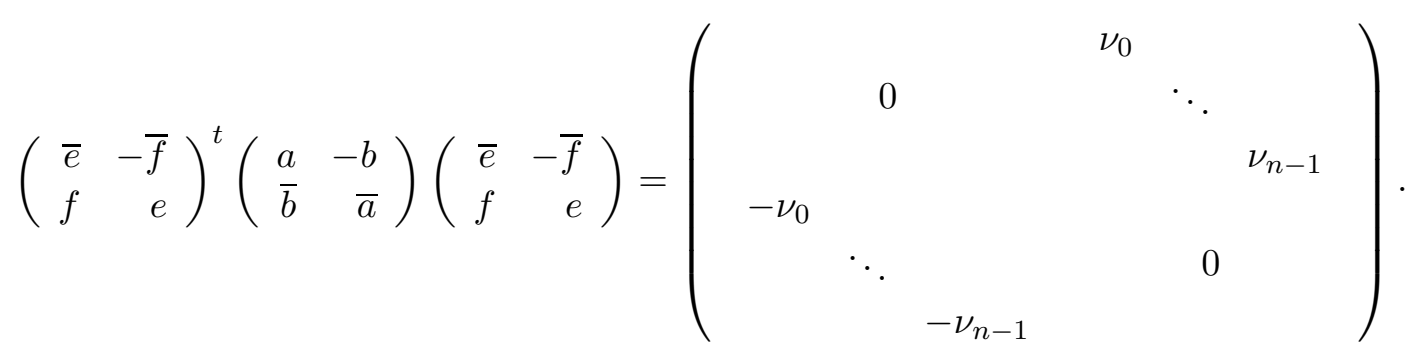

Now set $\mathcal{E}:=\bar{e}+\bar{f} \mathbf{j}$. The left hand side of $(2.22)$ is exactly $\tau(\mathcal{E})^{t} M \tau(\mathcal{E})$. We see that $\mathcal{E}$ is unitary since

$$
\bar{e}+\bar{f} \mathbf{j}=-\mathbf{j}(e+f \mathbf{j}) \mathbf{j},
$$

and $e+f \mathbf{j}$ and $\mathbf{j}$ are both unitary. So (1.6) holds. At last we find that

$$
\omega^{A}=\tau(\mathcal{E}) \cdot \widetilde{\omega}^{A}
$$

by $\tau(\mathcal{E}) \cdot \tau\left(\mathcal{E}^{*}\right) \cdot \omega^{A}=\tau\left(\mathcal{E} \mathcal{E}^{*}\right) \cdot \omega^{A}=\omega^{A}$ and

$$
\begin{aligned}
\omega & =\sum_{A, B=0}^{2 n-1} M_{A B} \tau(\mathcal{E}) \cdot \widetilde{\omega}^{A} \wedge \tau(\mathcal{E}) \cdot \widetilde{\omega}^{B}=\sum_{A, B, C, D} M_{A B} \tau(\mathcal{E})_{A C} \widetilde{\omega}^{C} \wedge \tau(\mathcal{E})_{B D} \widetilde{\omega}^{D} \\
& =\sum_{C, D}\left(\tau(\mathcal{E})^{t} M \tau(\mathcal{E})\right)_{C D} \widetilde{\omega}^{C} \wedge \widetilde{\omega}^{D},
\end{aligned}
$$

from which we get (1.7).

Proof of Theorem 1.2. In order to use results in the proof of Theorem 1.1, we use notations there. Let $\mathcal{M}=-b+a \mathbf{j}$ be a hyperhermitian matrix as above, and let $\omega=\sum_{A, B=0}^{2 n-1} M_{A B} \omega^{A} \wedge \omega^{B}$ be the 2-element, where $M=\left(M_{A B}\right)=\tau(\mathcal{M}) J$. Let $\mathcal{B}=e+f \mathbf{j}$ be the unitary matrix such that (2.19) holds. Then (1.7) holds with $\mathcal{E}:=\bar{e}+\bar{f} \mathbf{j}$ and $\widetilde{\omega}^{A}=\mathcal{E}^{*} \cdot \omega^{A}$.

Now apply Propositions 2.2 (2) to determinants of both sides of (2.19) to get

$$
\operatorname{det}(-b+a \mathbf{j})=\nu_{0} \cdots \nu_{n-1} .
$$

The $n$-th exterior products of both sides of (1.7) give us

$$
\omega \wedge \cdots \wedge \omega=2^{n} n ! \nu_{0} \cdots \nu_{n-1} \widetilde{\omega}^{0} \wedge \widetilde{\omega}^{n} \wedge \cdots \wedge \widetilde{\omega}^{n-1} \wedge \widetilde{\omega}^{2 n-1} .
$$

We claim that

$$
\widetilde{\omega}^{0} \wedge \widetilde{\omega}^{n} \cdots \wedge \widetilde{\omega}^{n-1} \wedge \widetilde{\omega}^{2 n-1}=\Omega_{2 n} .
$$

This is because $\tau\left(\mathcal{E}^{*}\right)$ is symplectic by Proposition 2.3 (3), since $\mathcal{E}=\bar{e}+\bar{f} \mathbf{j}$ is quaternionic unitary. So $\tau\left(\mathcal{E}^{*}\right) . \beta_{n}=\beta_{n}$, i.e.

$$
\sum_{l=0}^{n-1} \widetilde{\omega}^{l} \wedge \widetilde{\omega}^{l+n}=\sum_{l=0}^{n-1} \omega^{l} \wedge \omega^{l+n},
$$

as in (2.11), whose $n$-th exterior products give us (2.25).

Apply (2.25) to (2.24) to get

$$
\triangle_{n}(M, \cdots, M)=2^{n} n ! \operatorname{det}(-b+a \mathbf{j}) .
$$


by definition of $\triangle_{n}$ in (1.8). Namely for the 2 -form

$$
\omega_{\mathcal{M}}:=\sum_{A, B}[\tau(\mathcal{M}) J]_{A B} \omega^{A} \wedge \omega^{B}
$$

associated to a hyperhermitian $n \times n$-matrix $\mathcal{M}=-b+a \mathbf{j}$, we have

$$
\omega_{\mathcal{M}} \wedge \cdots \wedge \omega_{\mathcal{M}}=2^{n} n ! \operatorname{det}(\mathcal{M}) \Omega_{2 n}
$$

Applying (2.26) to $\mathcal{M}=\lambda_{1} \mathcal{M}_{1}+\cdots+\lambda_{n} \mathcal{M}_{n}$ for real numbers $\lambda_{1}, \cdots, \lambda_{n}$, which is also hyperhermitian since each $\mathcal{M}_{j}$ is, we get

$$
\left(\lambda_{1} \omega_{\mathcal{M}_{1}}+\cdots\right) \wedge \cdots \wedge\left(\lambda_{1} \omega_{\mathcal{M}_{1}}+\cdots\right)=2^{n} n ! \operatorname{det}\left(\lambda_{1} \tau\left(\mathcal{M}_{1}\right) J+\cdots+\lambda_{n} \tau\left(\mathcal{M}_{n}\right) J\right) \Omega_{2 n} .
$$

The coefficients of the monomial $\lambda_{1} \cdots \lambda_{n}$ gives us

$$
n ! \omega_{\mathcal{M}_{1}} \wedge \cdots \wedge \omega_{\mathcal{M}_{n}}=2^{n}(n !)^{2} \operatorname{det}\left(\mathcal{M}_{1}, \cdots, \mathcal{M}_{n}\right) \Omega_{2 n} .
$$

The theorem is proved.

3. The quaternionic Monge-Ampère operator and its fundamental solution

For a point $q=\left(\begin{array}{c}q_{0} \\ \vdots \\ q_{n-1}\end{array}\right) \in \mathbb{H}^{n}$, write

$$
q_{l}:=x_{4 l}+x_{4 l+1} \mathbf{i}+x_{4 l+2} \mathbf{j}+x_{4 l+3} \mathbf{k},
$$

$l=0, \ldots, n-1$. The Cauchy-Fueter operator is

$$
\frac{\partial}{\partial \overline{q_{l}}}=\partial_{x_{4 l}}+\mathbf{i} \partial_{x_{4 l+1}}+\mathbf{j} \partial_{x_{4 l+2}}+\mathbf{k} \partial_{x_{4 l+3}}
$$

and its conjugate

$$
\frac{\partial}{\partial q_{l}}:=\partial_{x_{4 l}}-\mathbf{i} \partial_{x_{4 l+1}}-\mathbf{j} \partial_{x_{4 l+2}}-\mathbf{k} \partial_{x_{4 l+3}} .
$$

The quaternionic Hessian of a real $C^{2}$ function $u$ at the point $q$ is the hyperhermitian matrix

$$
\left(\frac{\partial^{2} u}{\partial \overline{q_{l}} \partial q_{k}}(q)\right) \text {. }
$$

Proof of Theorem 1.3. Note that it follows from definitions of $\nabla_{A \alpha}$ 's in (1.13) and $\triangle_{A B}$ 's in (1.12) that

$$
\mathbf{j} \nabla_{(n+k) 0^{\prime}}=-\nabla_{k 1^{\prime} \mathbf{j},}, \quad \mathbf{j} \nabla_{(n+k) 1^{\prime}}=\nabla_{k 0^{\prime}} \mathbf{j},
$$

and

$$
\begin{aligned}
\frac{\partial^{2} u}{\partial \overline{q_{l}} \partial q_{k}} & =\left(\frac{\partial}{\partial x_{4 l}}+\mathbf{i} \frac{\partial}{\partial x_{4 l+1}}+\mathbf{j} \frac{\partial}{\partial x_{4 l+2}}+\mathbf{k} \frac{\partial}{\partial x_{4 l+3}}\right)\left(\frac{\partial}{\partial x_{4 k}}-\mathbf{i} \frac{\partial}{\partial x_{4 k+1}}-\mathbf{j} \frac{\partial}{\partial x_{4 k+2}}-\mathbf{k} \frac{\partial}{\partial x_{4 k+3}}\right) u \\
& =\left(\nabla_{l 0^{\prime}}-\nabla_{l 1^{\prime}} \mathbf{j}\right)\left(\nabla_{(n+k) 1^{\prime}}-\mathbf{j} \nabla_{(n+k) 0^{\prime}}\right) u \\
& =\left(\nabla_{l 0^{\prime}} \nabla_{(n+k) 1^{\prime}}-\nabla_{l 1^{\prime}} \nabla_{(n+k) 0^{\prime}}\right) u+\left(\nabla_{l 0^{\prime}} \nabla_{k 1^{\prime}}-\nabla_{l 1^{\prime}} \nabla_{k 0^{\prime}}\right) u \mathbf{j} \\
& =2\left(\triangle_{l(n+k)} u+\triangle_{l k} u \mathbf{j}\right)
\end{aligned}
$$


for $l, k=0, \ldots, n-1$. So the the quaternionic Hessian can be written as

$$
\left(\frac{\partial^{2} u}{\partial \overline{q_{l}} \partial q_{k}}\right)=a+b \mathbf{j}
$$

with $n \times n$ complex matrices

$$
a:=2\left(\triangle_{l(n+k)} u\right), \quad b:=2\left(\triangle_{l k} u\right)
$$

Thus

$$
\tau\left(\frac{\partial^{2} u}{\partial \overline{q_{l}} \partial q_{k}}\right) J=\left(\begin{array}{rr}
a & -b \\
\bar{b} & \bar{a}
\end{array}\right) J=\left(\begin{array}{rr}
b & a \\
-\bar{a} & \bar{b}
\end{array}\right)=2\left(\begin{array}{rr}
\triangle_{l k} u & \triangle_{l(n+k)} u \\
-\triangle_{l(n+k)} u & \frac{\triangle_{l k} u}{}
\end{array}\right) .
$$

We find that

$$
\tau\left(\frac{\partial^{2} u}{\partial \overline{q_{l}} \partial q_{k}}\right) J=2\left(\triangle_{A B} u\right)
$$

because

$$
\overline{\triangle_{l(n+k)} u}=\overline{\nabla_{l 0^{\prime}} \nabla_{(n+k) 1^{\prime}} u}-\overline{\nabla_{l 1^{\prime}} \nabla_{(n+k) 0^{\prime}} u}=\nabla_{(n+l) 1^{\prime}} \nabla_{k 0^{\prime}} u-\nabla_{(n+l) 0^{\prime}} \nabla_{k 1^{\prime}} u=-\triangle_{(n+l) k} u
$$

by definition of $\nabla_{A \alpha}$ 's in (1.13). Similarly,

$$
\overline{\triangle_{l k} u}=\triangle_{(n+l)(n+k)} u .
$$

The result follows from (3.5) by applying Theorem 1.2 to $\mathcal{M}_{j}=\left(\frac{\partial^{2} u_{j}}{\partial \overline{q_{l}} \partial q_{k}}\right)$.

The first-order differential operators $\nabla_{A \alpha}$ 's in (1.13) are again the relabelling of the following differential operators we used in [15]:

$$
\left(\begin{array}{cc}
\nabla_{00^{\prime}} & \nabla_{01^{\prime}} \\
\nabla_{10^{\prime}} & \nabla_{11^{\prime}} \\
\vdots & \vdots \\
\nabla_{(2 l) 0^{\prime}} & \nabla_{(2 l) 1^{\prime}} \\
\nabla_{(2 l+1) 0^{\prime}} & \nabla_{(2 l+1) 1^{\prime}} \\
\vdots & \vdots
\end{array}\right):=\left(\begin{array}{cc}
\partial_{x_{0}}+\mathbf{i} \partial_{x_{1}} & -\partial_{x_{2}}-\mathbf{i} \partial_{x_{3}} \\
\partial_{x_{2}}-\mathbf{i} \partial_{x_{3}} & \partial_{x_{0}}-\mathbf{i} \partial_{x_{1}} \\
\vdots & \vdots \\
\partial_{x_{4 l}}+\mathbf{i} \partial_{x_{4 l+1}} & -\partial_{x_{4 l+2}}-\mathbf{i} \partial_{x_{4 l+3}} \\
\partial_{x_{4 l+2}}-\mathbf{i} \partial_{x_{4 l+3}} & \partial_{x_{4 l}}-\mathbf{i} \partial_{x_{4 l+1}} \\
\vdots & \vdots
\end{array}\right)
$$

Let $\Omega$ be a domain in $\mathbb{H}^{n}$. Recall that $d_{0}, d_{1}: C_{0}^{\infty}\left(\Omega, \wedge^{p} \mathbb{C}^{2 n}\right) \rightarrow C_{0}^{\infty}\left(\Omega, \wedge^{p+1} \mathbb{C}^{2 n}\right)$ are given by

$$
\begin{aligned}
& d_{0} F:=\sum_{I} \sum_{A=0}^{2 n-1} \nabla_{A 0^{\prime}} f_{I} \omega^{A} \wedge \omega^{I}, \\
& d_{1} F:=\sum_{I} \sum_{A=0}^{2 n-1} \nabla_{A 1^{\prime}} f_{I} \omega^{A} \wedge \omega^{I},
\end{aligned}
$$

for $F=\sum_{I} f_{I} \omega^{I} \in C_{0}^{\infty}\left(\Omega, \wedge^{p} \mathbb{C}^{2 n}\right)$, where $\omega^{I}:=\omega^{i_{1}} \wedge \ldots \wedge \omega^{i_{p}}$ for the multi-index $I=\left(i_{1}, \ldots, i_{p}\right)$. We say $F$ is closed if

$$
d_{0} F=d_{1} F=0
$$

Then we have

$$
\triangle u=d_{0} d_{1} u
$$


Proposition 3.1. (Proposition 2.2 in [15]) (1) $d_{0} d_{1}=-d_{1} d_{0}$.

(2) $d_{0}^{2}=d_{1}^{2}=0$.

(3) For $F \in C_{0}^{\infty}\left(\Omega, \wedge^{p} \mathbb{C}^{2 n}\right), G \in C_{0}^{\infty}\left(\Omega, \wedge^{q} \mathbb{C}^{2 n}\right)$, we have

$$
d_{\alpha}(F \wedge G)=d_{\alpha} F \wedge G+(-1)^{p} F \wedge d_{\alpha} G, \quad \alpha=0,1 .
$$

$z^{A \alpha}$ 's given by

$$
\left(\begin{array}{cc}
z^{00^{\prime}} & z^{01^{\prime}} \\
\vdots & \vdots \\
z^{(l) 0^{\prime}} & z^{(l) 1^{\prime}} \\
\vdots & \vdots \\
z^{n 0^{\prime}} & z^{n 1^{\prime}} \\
\vdots & \vdots \\
z^{(n+l) 0^{\prime}} & z^{(n+l) 1^{\prime}} \\
\vdots & \vdots
\end{array}\right):=\left(\begin{array}{cc}
x_{0}-\mathbf{i} x_{1} & -x_{2}+\mathbf{i} x_{3} \\
\vdots & \vdots \\
x_{4 l}-\mathbf{i} x_{4 l+1} & -x_{4 l+2}+\mathbf{i} x_{4 l+3} \\
\vdots & \vdots \\
x_{2}+\mathbf{i} x_{3} & x_{0}+\mathbf{i} x_{1} \\
\vdots & \vdots \\
x_{4 l+2}+\mathbf{i} x_{4 l+3} & x_{4 l}+\mathbf{i} x_{4 l+1} \\
\vdots & \vdots
\end{array}\right)
$$

can be viewed as independent variables formally and $\nabla_{A \alpha}$ 's are derivatives with respect to these variables by the following lemma.

Lemma 3.1. (Lemma 3.1 and Corollary 3.1 (1) in [15]) (1) $\nabla_{A \alpha} z^{B \beta}=2 \delta_{A}^{B} \delta_{\alpha}^{\beta}$.

(2) $\nabla_{A \alpha}\left(\|q\|^{2}\right)=2 \overline{z^{A \alpha}}$.

The function $-\frac{1}{\|q\|^{2}}$ is a PSH function (Proposition 4.1 in [15]), and can be shown to be the fundamental solution of the quaternionic Monge-Ampère operator in the following simple way by using $d_{0}$ and $d_{1}$.

Proposition 3.2. (Proposition 4.1 in [15]) $\left(\triangle\left(-\frac{1}{\|q\|^{2}}\right)\right)^{n}=\frac{8^{n} n ! \pi^{2 n}}{(2 n) !} \delta_{0} \Omega_{2 n}$.

Proof. Note that

$$
d_{\alpha}\left(-\frac{1}{\|q\|^{2}+\varepsilon}\right)=\frac{d_{\alpha}\|q\|^{2}}{\left(\|q\|^{2}+\varepsilon\right)^{2}}
$$

and so

$$
\triangle\left(-\frac{1}{\|q\|^{2}+\varepsilon}\right)=d_{0} d_{1}\left(-\frac{1}{\|q\|^{2}+\varepsilon}\right)=-\frac{2 d_{0}\|q\|^{2} \wedge d_{1}\|q\|^{2}}{\left(\|q\|^{2}+\varepsilon\right)^{3}}+\frac{d_{0} d_{1}\|q\|^{2}}{\left(\|q\|^{2}+\varepsilon\right)^{2}}
$$

where

$$
d_{\alpha}\|q\|^{2}=2 \sum_{A=0}^{2 n-1} \overline{z^{A \alpha}} \omega^{A},
$$

by using Lemma 3.1 (2). In particular, we have

$$
d_{1}\|q\|^{2}=2 \sum_{l=0}^{n-1}\left(-z^{(n+l) 0^{\prime}} \omega^{l}+z^{l 0^{\prime}} \omega^{n+l}\right),
$$

and so

$$
d_{0} d_{1}\|q\|^{2}=8 \beta_{n}
$$


by using Lemma 3.1 (1). It follows that

$$
\left(\triangle\left(-\frac{1}{\|q\|^{2}+\varepsilon}\right)\right)^{n}=-\frac{2 \cdot 8^{n-1} n}{\left(\|q\|^{2}+\varepsilon\right)^{2 n+1}} d_{0}\|q\|^{2} \wedge d_{1}\|q\|^{2} \wedge \beta_{n}^{n-1}+\frac{8^{n}}{\left(\|q\|^{2}+\varepsilon\right)^{2 n}} \beta_{n}^{n}
$$

by $\omega \wedge \omega=0$ for any 1 -form $\omega$ and (3.10). Note that $\beta_{n}^{n}=n ! \Omega_{2 n}$ and

$$
z^{l 0^{\prime}} z^{(n+l) 1^{\prime}}-z^{(n+l) 0^{\prime}} z^{l 1^{\prime}}=\left|q_{l}\right|^{2},
$$

from which we see that

$$
d_{0}\|q\|^{2} \wedge d_{1}\|q\|^{2}=4 \sum_{l=0}^{n-1}\left|q_{l}\right|^{2} \omega^{l} \wedge \omega^{n+l}+\sum_{|j-k| \neq n} a_{j k} \omega^{j} \wedge \omega^{k} .
$$

Substitute (3.12) into (3.11) to get

$$
\left(\triangle\left(-\frac{1}{\|q\|^{2}+\varepsilon}\right)\right)^{n}=\frac{8^{n} n ! \varepsilon}{\left(\|q\|^{2}+\varepsilon\right)^{2 n+1}} \Omega_{2 n}
$$

Let $\varepsilon=0$, we get

$$
\left(\triangle\left(-\frac{1}{\|q\|^{2}}\right)\right)^{n}=0
$$

for $q \neq 0$. Then we can check $\left(\triangle\left(-\frac{1}{\|q\|^{2}}\right)\right)^{n}=\frac{8^{n} n ! \pi^{2 n}}{(2 n) !} \delta_{0} \Omega_{2 n}$ by integrating (3.13) as in the proof of Proposition 4.1 in [15].

\section{THE INVARIANCE OF $d_{\alpha}$ AND $\triangle$ UNDER QUATERNIONIC LINEAR TRANSFORMATIONS}

The operators $d_{0}$ and $d_{1}$ in (3.7) depend on the choice of the coordinates $x_{j}$ 's and the basis $\left\{\omega^{A}\right\}$. Let us give their transformation formula.

For a quaternionic vector $q=\left(\begin{array}{l}q_{0} \\ \vdots \\ q_{n-1}\end{array}\right) \in \mathbb{H}^{n}$, write

$$
q=x^{(0)}+\mathbf{i} x^{(1)}+\mathbf{j} x^{(2)}+\mathbf{k} x^{(3)}, \quad \text { with } \quad x^{(\beta)}=\left(\begin{array}{c}
\vdots \\
x_{4 l+\beta} \\
\vdots
\end{array}\right) \in \mathbb{R}^{n},
$$

$\beta=0,1,2,3$, where $x_{0}, \ldots, x_{4 n-1}$ are real coordinates of $q$ given by (3.1). A quaternionic matrix $\mathcal{U} \in M_{\mathbb{H}}(n, n)$ defines a right linear transformation $\mathbb{H}^{4 n} \rightarrow \mathbb{H}^{4 n}$ given by $\widetilde{q}=\mathcal{U} q$. Write

$$
\mathcal{U}=U_{0}+\mathbf{i} U_{1}+\mathbf{j} U_{2}+\mathbf{k} U_{3},
$$

for some real $(n \times n)$-matrices $U_{0}, U_{1}, U_{2}, U_{3}$. If we denote

$$
\mathcal{U}^{\mathbb{R}}:=\left(\begin{array}{rrrr}
U_{0} & -U_{1} & -U_{2} & -U_{3} \\
U_{1} & U_{0} & -U_{3} & U_{2} \\
U_{2} & U_{3} & U_{0} & -U_{1} \\
U_{3} & -U_{2} & U_{1} & U_{0}
\end{array}\right), \quad q^{\mathbb{R}}:=\left(\begin{array}{c}
x^{(0)} \\
x^{(1)} \\
x^{(2)} \\
x^{(3)}
\end{array}\right) \in \mathbb{R}^{4 n}
$$

it is direct to check that

$$
(\mathcal{U} q)^{\mathbb{R}}=\mathcal{U}^{\mathbb{R}} q^{\mathbb{R}}
$$

(cf. (2.9)-(2.10) in [18]). 
Proposition 4.1. Let $\widetilde{\nabla}_{A \alpha}$ be the operators defined by (1.13) in terms of the real coordinates $\widetilde{x}$ of $\widetilde{q}=\mathcal{U} q$, i.e. $\widetilde{q}_{l}=\widetilde{x}_{4 l}+\boldsymbol{i} \widetilde{x}_{4 l+1}+\widetilde{j}_{4 l+2}+\boldsymbol{k} \widetilde{x}_{4 l+3}$. Under the transformation $\mathcal{U}$, write $u(q):=\widetilde{u}(\mathcal{U} q)$. Then we have

$$
\left.\nabla_{A \alpha} u\right|_{q}=\left.\sum_{B=0}^{2 n-1} \overline{\tau(\mathcal{U})}_{B A} \widetilde{\nabla}_{B \alpha} \widetilde{u}\right|_{\mathcal{U} q},
$$

for $\alpha=0^{\prime}, 1^{\prime}, A=0, \ldots, 2 n-1$.

Proof. In this proof, let $\hat{x}_{a}$ be the $a$-th entry of the vector $q^{\mathbb{R}}$ in (4.3). It is just relabeling of coordinates $\left(x_{0}, \ldots, x_{4 n-1}\right)$. Similarly, $\hat{\widetilde{x}}_{a}$ be the $a$-th entry of the corresponding vector $\widetilde{q}^{\mathbb{R}}$ for $\widetilde{q}$. By definition, we have

$$
\partial_{\hat{x}_{a}}\left[\widetilde{u}\left(\mathcal{U}^{\mathbb{R}} q^{\mathbb{R}}\right)\right]=\sum_{b=0}^{4 n-1}\left(\mathcal{U}^{\mathbb{R}}\right)_{b a}\left(\partial_{\widehat{\widetilde{x}}_{b}} \widetilde{u}\right)(\mathcal{U} q) .
$$

Note that $x_{j}^{(0)}=\hat{x}_{j}, x_{j}^{(1)}=\hat{x}_{n+j}$, for $j=0, \ldots, n-1$. Therefore, we have

$$
\begin{aligned}
\nabla_{j 0^{\prime}} u= & \partial_{x_{j}^{(0)}} u+\mathbf{i} \partial_{x_{j}^{(1)}} u=\sum_{b=0}^{4 n-1}\left\{\left(\mathcal{U}^{\mathbb{R}}\right)_{b j} \partial_{\widetilde{x}_{b}}+\mathbf{i}\left(\mathcal{U}^{\mathbb{R}}\right)_{b(n+j)} \partial_{\widetilde{x}_{b}}\right\} \widetilde{u} \\
= & \sum_{l=0}^{n-1}\left\{\left(U_{0}\right)_{l j} \partial_{\widetilde{x}_{4 l}}+\left(U_{1}\right)_{l j} \partial_{\widetilde{x}_{4 l+1}}+\left(U_{2}\right)_{l j} \partial_{\widetilde{x}_{4 l+2}}+\left(U_{3}\right)_{l j} \partial_{\widetilde{x}_{4 l+3}}\right\} \widetilde{u} \\
& +\mathbf{i} \sum_{l=0}^{n-1}\left\{-\left(U_{1}\right)_{l j} \partial_{\widetilde{x}_{4 l}}+\left(U_{0}\right)_{l j} \partial_{\widetilde{x}_{4 l+1}}+\left(U_{3}\right)_{l j} \partial_{\widetilde{x}_{4 l+2}}-\left(U_{2}\right)_{l j} \partial_{\widetilde{x}_{4 l+3}}\right\} \widetilde{u} \\
= & \sum_{l=0}^{n-1}\left\{\left(U_{0}-\mathbf{i} U_{1}\right)_{l j}\left(\partial_{\widetilde{x}_{4 l}}+\mathbf{i} \partial_{\widetilde{x}_{4 l+1}}\right)+\left(U_{2}+\mathbf{i} U_{3}\right)_{l j}\left(\partial_{\widetilde{x}_{4 l+2}}-\mathbf{i} \partial_{\widetilde{x}_{4 l+3}}\right)\right\} \widetilde{u} \\
= & \sum_{l=0}^{n-1}\left\{\left(U_{0}-\mathbf{i} U_{1}\right)_{l j} \widetilde{\nabla}_{l 0^{\prime}}+\left(U_{2}+\mathbf{i} U_{3}\right)_{l j} \widetilde{\nabla}_{(n+l) 0^{\prime}}\right\} \widetilde{u}
\end{aligned}
$$

by the first two columns of $\mathcal{U}^{\mathbb{R}}$ in (4.3) and $\nabla_{j \alpha}$ 's in (1.13). Similarly, we have

$$
\begin{aligned}
\nabla_{(n+j) 0^{\prime}} u & =\partial_{x_{j}^{(2)}} u-\mathbf{i} \partial_{x_{j}^{(3)}} u \\
& =\sum_{l=0}^{n-1}\left\{\left(-U_{2}+\mathbf{i} U_{3}\right)_{l j}\left(\partial_{\widetilde{x}_{4 l}}+\mathbf{i} \partial_{\widetilde{x}_{4 l+1}}\right)+\left(U_{0}+\mathbf{i} U_{1}\right)_{l j}\left(\partial_{\widetilde{x}_{4 l+2}}-\mathbf{i} \partial_{\widetilde{x}_{4 l+3}}\right)\right\} \widetilde{u} .
\end{aligned}
$$

Note that

$$
\overline{\tau(\mathcal{U})}:=\left(\begin{array}{rr}
U_{0}-\mathbf{i} U_{1} & -U_{2}+\mathbf{i} U_{3} \\
U_{2}+\mathbf{i} U_{3} & U_{0}+\mathbf{i} U_{1}
\end{array}\right) .
$$

It follows from (4.6)-(4.7) that (4.5) holds for $\alpha=0^{\prime}$. Then (4.5) for $\alpha=1^{\prime}$ follows by taking conjugate on both sides of (4.6)-(4.7) and noting that $\overline{\nabla_{j 0^{\prime}}}=\nabla_{(n+j) 1^{\prime}}, \overline{\nabla_{(n+j) 0^{\prime}}}=-\nabla_{j 1^{\prime}}$ and so for $\widetilde{\nabla}$.

For a basis $\left\{\omega^{0}, \omega^{1}, \ldots, \omega^{2 n-1}\right\}$ of $\mathbb{C}^{2 n},\left\{\widetilde{\omega}^{0}, \widetilde{\omega}^{1}, \ldots, \widetilde{\omega}^{2 n-1}\right\}$ given by

$$
\widetilde{\omega}^{A}:=\overline{\tau(\mathcal{U})} \cdot \omega^{A}=\sum_{B=0}^{2 n-1} \overline{\tau(\mathcal{U})}_{A B} \omega^{B}
$$


is also a basis for $\mathcal{U} \in \mathrm{GL}_{\mathbb{H}}(n)$.

Proposition 4.2. (Proposition 2.4 in [15]) Let $u$ be a real $C^{1}$ functions. Then $d_{\alpha} u$ is invariant under a quaternionic linear transformations $\mathcal{U}$ on $\mathbb{H}^{n}$, i.e.,

$$
\left.d_{\alpha} u\right|_{q}=\left.\widetilde{d_{\alpha}} \widetilde{u}\right|_{\mathcal{U} q}
$$

for $\alpha=0^{\prime}, 1^{\prime}$, where $u(q):=\widetilde{u}(\mathcal{U} q), \widetilde{d_{\alpha}} \widetilde{u}=\sum_{A} \widetilde{\nabla}_{A \alpha} \widetilde{u} \widetilde{\omega}^{A}$.

Proof. By Proposition 4.1, we get

$$
d_{\alpha} u=\sum_{A} \nabla_{A \alpha} u \omega^{A}=\sum_{A, B} \overline{\tau(\mathcal{U})}_{B A} \widetilde{\nabla}_{B \alpha} \widetilde{u} \omega^{A}=\sum_{B} \widetilde{\nabla}_{B \alpha} \widetilde{u} \widetilde{\omega}^{B}=\widetilde{d}_{\alpha} \widetilde{u} .
$$

The transformation formula follows.

Corollary 4.1. (Corollary 2.2 in [15]) $\triangle u$ and $(\triangle u)^{n}$ are also invariant under quaternionic linear transformations on $\mathbb{H}^{n}$, i.e., $\left.\triangle u\right|_{q}=\left.\widetilde{\triangle} \widetilde{u}\right|_{\mathcal{U} q}$.

Proof. We have

$$
\begin{aligned}
\triangle u(q) & =d_{0} d_{1} u(q)=d_{0}\left[\left(\widetilde{d_{1}} \widetilde{u}\right)(\mathcal{U} q)\right]=\sum_{A, B} \nabla_{A 0^{\prime}}\left[\left(\widetilde{\nabla}_{B 1^{\prime}} \widetilde{u}\right)(\mathcal{U} q)\right] \omega^{A} \wedge \widetilde{\omega}^{B} \\
& =\sum_{A, B, C} \overline{\tau(\mathcal{U})}_{C A}\left(\widetilde{\nabla}_{C 0^{\prime}} \widetilde{\nabla}_{B 1^{\prime}} \widetilde{u}\right)(\mathcal{U} q) \omega^{A} \wedge \widetilde{\omega}^{B}=\sum_{B, C}\left(\widetilde{\nabla}_{C 0^{\prime}} \widetilde{\nabla}_{B 1^{\prime}} \widetilde{u}\right)(\mathcal{U} q) \widetilde{\omega}^{C} \wedge \widetilde{\omega}^{B} \\
& =(\widetilde{\triangle} \widetilde{u})(\mathcal{U} q)
\end{aligned}
$$

by applying Proposition $4.1,4.2$.

Hence $\triangle_{n} u$ is also invariant under quaternionic unitary transformation $\mathcal{U}$ by $\mathcal{U} . \Omega_{2 n}=\Omega_{2 n}$, i.e.

$$
\triangle_{n}\left(u_{1}, \ldots, u_{n}\right)(q)=\widetilde{\triangle}_{n}\left(\widetilde{u}_{1}, \ldots, \widetilde{u}_{n}\right)(\mathcal{U} q) .
$$

\section{REFERENCES}

[1] S. Alesker, Non-commmutative linear algebra and plurisubharmonic functions of quaternionic variables, Bull. Sci. Math. 127(1) (2003), 1-35.

[2] S. Alesker, Quaternionic Monge-Ampère equations, J. Geom. Anal. 13 (2003), no. 2, 205-238.

[3] S. Alesker, Pluripotential theory on quaternionic manifolds, J. Geom. Phys. 62 (2012), no. 5, 1189-1206.

[4] S. Alesker and E. Shelukhin, On a uniform estimate for the quaternionic Calabi problem, Israel J. Math. 197, (2013), 309-327.

[5] S. Alesker and E. Shelukhin, A uniform estimate for general quaternionic Calabi problem (with appendix by Daniel Barlet), Adv. in Math. 316 (2017), 1-52.

[6] S. Alesker And M. Verbitsky, Plurisubharmonic functions on hypercomplex manifolds and HKTgeometry, J. Geom. Anal. 16 (2006), 375-399.

[7] R. J. Baston, Quaternionic complexes, J. Geom. Phys. 8 (1992), no. 1-4, 29-52.

[8] E. Bedford ANd B.A. TAYlor, A new capacity for plurisubharmonic functions, Acta Math. 149 (1982), no. 1-2, 1-40.

[9] J. Bureš And V. Souček, Complexes of invariant differential operators in several quaternionic variables, Complex Var. Elliptic Equ. 51 (2006), no. 5-6, 463-487.

[10] R. Harvey and H. Lawson, An introduction to potential theory in calibrated geometry, Amer. J. Math. 131 (2009), 893-944.

[11] R. HaRvey and H. Lawson, Duality of positive currents and plurisubharmonic functions in calibrated geometry, Amer. J. Math. 131 (2009), 1211-1239. 
[12] M. KlimeK, Pluripotential Theory, Clarendon Press (1991).

[13] D. WAN, The continuity and range of the quaternionic Monge-Ampère operator on quaternionic space, Math. Z. 285 (2017), 461-478.

[14] D. WAn And Q. KAng, Potential theory for quaternionic plurisubharmonic functions, Michigan Math. J. 66 (2017), no. 1, 3-20.

[15] D. WAn AND W. WANG, On quaternionic Monge-Ampère operator, closed positive currents and LelongJensen type formula on the quaternionic space, Bull. Sci. Math. 141 (2017), 267-311.

[16] D. WAn And W. Zhang, Quasicontinuity and maximality of quaternionic plurisubharmonic functions, $J$. Math. Anal. Appl. 424 (2015), 86-103.

[17] W. WAng, The $k$-Cauchy-Fueter complex, Penrose transformation and Hartogs' phenomenon for quaternionic k-regular functions, J. Geom. Phys. 60 (2010), 513-530.

[18] W. Wang, On the optimal control method in quaternionic analysis, Bull. Sci. Math. 135 (2011), no. 8, 988-1010.

[19] W. Wang, On quaternionic complexes over unimodular quaternionic manifolds, Diff. Geom. Appl. 58 (2018), 227-253.

[20] J. ZHu, Dirichlet problem of quaternionic Monge-Ampr̀e equations, Israel J. Math. 214 (2016), 597-619. 